Supporting Information

\title{
Nanoscale Covalent Organic Frameworks with Donor-Acceptor Structure for Enhanced Photothermal Ablation of Tumors
}

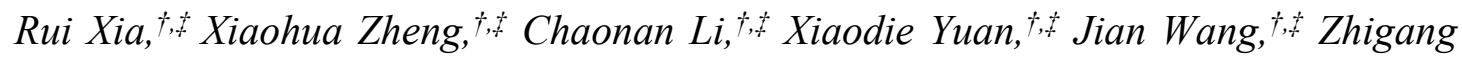
Xie $e^{*,+, t}$ and Xiabin Jing ${ }^{\dagger}$

†State Key Laboratory of Polymer Physics and Chemistry, Changchun Institute of Applied Chemistry, Chinese Academy of Sciences, Changchun, Jilin 130022, P. R. China

University of Science and Technology of China, Hefei 230026, P. R. China 


\section{Table of Contents}

\section{Experimental section.}

Figure S1. Transmission electron microscopy images of DPPN COF with different sizes $800 \mathrm{~nm}(\mathrm{a}), 600 \mathrm{~nm}$ (b) and $400 \mathrm{~nm}$ (c).

Figure S2. TEM images of DPPN COF reacted for $1 \mathrm{~h} \mathrm{(a),} 4 \mathrm{~h} \mathrm{(b),} 12 \mathrm{~h} \mathrm{(c)} \mathrm{and} 24 \mathrm{~h}$ (d).

Figure S3. TEM images (a), UV absorption spectrum (b) and size distribution (c) of DPPN COF prepared in benzonitrile.

Figure S4. TEM images of DPPN COF using dioxane/ methanol (a) or acetone/ methanol (b) as solvents.

Figure S5. TEM images (a), UV absorption spectrum (b) of DPPN COF using chloroform/ethanol as solvent.

Figure S6. TEM images of DPPC COF with diameters of $800 \mathrm{~nm}$ (a) and $400 \mathrm{~nm}$ (b). Figure S7. TEM images of DPPB COF with diameters of $800 \mathrm{~nm}$ (a) and $400 \mathrm{~nm}$ (b).

Figure S8. UV absorption spectra of DPPN COF from 500 to $1200 \mathrm{~nm}$ dispersed in THF.

Figure S9. Schematic illustration of construction units and optimized spatial configurations of units of DPPC, DPPB and DPPN COF by DFT calculations. Carbon (grey), Nitrogen (blue), Oxygen (red) and Hydrogen (white).

Figure S10. FT-IR spectra of DPPC COF and DPPB COF and their corresponding building blocks.

Figure S11. Transmission electron microscopy images of DPPN COF with $300 \mathrm{~nm}$ diameter dispersed in THF (a). Transmission electron microscopy images of DPPN COF with $300 \mathrm{~nm}$ diameter assembled with $\mathrm{MPEG}_{2000}$-DSPE in water (b).

Figure S12. Images of DPP monomer dissolved in THF and COFs dispersed in THF.

Figure S13. Images of DPPN COF before (left) or after (right) assembled with $\mathrm{MPEG}_{2000}$-DSPE dispersed in water.

Figure S14. Zeta potential of DPPC, DPPB and DPPN COF dispersed in water

Figure S15. Colloidal stability of COFs dispersed in water for 7 days. 
Figure S16. Colloidal stability of COFs dispersed in pH 6.5 PBS for 7 days.

Figure S17. The relationship between cooling period of time and the negative natural logarithm of temperature of DPPN COF.

Figure S18. Photodynamic effect of DPPC COF (a) and DPPB COF (b) detected by ABDA under $808 \mathrm{~nm}$ laser irradiation.

Figure S19. Thermal images of DPPC COF and DPPB COF with $808 \mathrm{~nm}$ laser irradiation at an intensity of $1 \mathrm{~W} / \mathrm{cm}^{2}$ in $5 \mathrm{~min}$.

Figure S20. Molecular structure, UV absorbance spectrum and fluorescence spectrum of BDP with green fluorescence (Excited by $450 \mathrm{~nm}$ wavelength).

Figure S21. Endocytosis contrast of DPPN COF/BDP at different time points and temperature (a). Fluorescence intensity comparison of DPPN COF/BDP (b). Scale bars, $20 \mu \mathrm{m}$

Figure S22. Cytotoxicity of A549 cells (a) treated with DPPC, DPPB, and DPPN COF without laser irradiation. Cytotoxicity of A549 cells (b) and treated with DPPC, DPPB, and DPPN COF with $808 \mathrm{~nm}$ laser $\left(0.8 \mathrm{~W} / \mathrm{cm}^{2}\right)$ irradiation for $5 \mathrm{~min}$.

Figure S23. Cell apoptosis assay of HeLa cells treated with PBS or COFs without laser irradiation.

Figure S24. Molecular structure (a), UV absorbance spectrum (b) and fluorescence spectrum (c) of NIR BDP with red fluorescence (Excited by $670 \mathrm{~nm}$ wavelength).

Figure S25. Fluorescence imaging of mice after intravenous injection from 0 to $24 \mathrm{~h}$ (a). Fluorescence intensity statistics of tumor at different points in time (b).

Figure S26. Temperature plots of two groups of mice with laser irradiation for $10 \mathrm{~min}$ at different point of time.

Figure S27. Corresponding images of tumors of four groups of mice after treatment for 14 days, $n=4$.

Figure S28. The corresponding weight changes of four groups of mice in 14 days.

Figure S29. H\&E staining of different organs of groups of mice with laser irradiation. Scale bars, $100 \mu \mathrm{m}$. 
Figure S30. UREA, ALT, UA, CREA and AST levels in the blood of groups of mice with laser irradiation.

Figure S31. ${ }^{1} \mathrm{H}$ NMR spectrum of DPP in $\mathrm{CDCl}_{3}$.

Table S1. Hydrodynamic size and polydispersity index (PDI) of DPPC, DPPB and DPPN COF dispersed in water.

Physical measurements: UV-Vis absorption spectra was tested with a Persee TU-1901 UV/Vis spectrophotometer. Fluorescence spectra of target materials were characterized using a PerkinElmer LS-55 Spectro fluorophotometer. FT-IR spectra was conducted on a Nicolet Impact 410 spectrometer. Morphologies of obtained COFs were measured by a JEOL JEM-1011 electron microscope operating at an acceleration voltage of $100 \mathrm{kV}$. CLSM (confocal laser scanning microscopy) images were taken using a Zeiss LSM 700 (Zurich, Switzerland). Nitrogen adsorption isotherms were tested on a Micromeritics ASAP 2010 analyzer. Powder X-ray diffraction (PXRD) was conducted on D8 Focus diffractometer (Bruker).

Singlet oxygen $\left({ }^{1} \mathrm{O}_{2}\right)$ detection: The ${ }^{1} \mathrm{O}_{2}$ generation of $\mathrm{COFs}$ was evaluated 9,10-Anthracenediyl-bis(methylene)-dimalonic acid (ABDA). All compounds (COFs and ABDA) were dissolved in water. Then the cuvette was exposed to laser irradiation $\left(808 \mathrm{~nm}, 0.5 \mathrm{~W} / \mathrm{cm}^{2}\right)$ for different time (every $60 \mathrm{~s}$ from 0 to $5 \mathrm{~min}$ ), and the mixed absorption spectra were recorded.

Photothermal activity of COFs: A $808 \mathrm{~nm}$ laser resource was employed to evaluate the photothermal activity of COFs dispered in water. Concentration and laser intensity-dependent properties of COFs during the measurements of the photothermal 
conversion process were deeply studied. MilliQ water was irradiated with the same condition as a control. All the photothermal data were recorded by a secure digital (SD) card installed in a thermocouple probe every $10 \mathrm{~s}$. The photothermal conversion efficiency of DPPN COF was caculated following the approach we reported earlier. ${ }^{[1]}$

Cell viability assays: The cytotoxicity of COFs was examined via MTT method. HeLa and A549 cells harvested in a logarithmic growth phase were seeded in 96-well plates at an initial density of $2 \times 10^{3}$ cells/well and incubated in $100 \mu \mathrm{L}$ DMEM at 37 ${ }^{\circ} \mathrm{C}$ in $5 \% \mathrm{CO} 2$ atmosphere for overnight. After removing the incubation medium, COF dispersions diluted with cell culture media to the desired concentration (50 to $150 \mu \mathrm{g} / \mathrm{mL}$ ) were added to cell wells. After incubation for 6 hours, they were exposed to $808 \mathrm{~nm}$ laser $\left(0.8 \mathrm{~W} / \mathrm{cm}^{2}\right)$ for 5 min then continued to incubate for 24 hours. $20 \mu \mathrm{L}$ MTT was added for each well. After $4 \mathrm{~h}$ incubation, culture medium was moved away and dimethyl sulfoxide (DMSO, $150 \mu \mathrm{L}$ ) was added. Cell viabilities were detected by the microplate reader.

Calcein-AM/PI examinations: To further test the cytotoxicity of COFs, HeLa cells were stained with the calcein-AM/propidium iodide (PI) to identify dead (red) and live (green) cells. Briefly, Hela cells incubated with culture medium or COFs $(150 \mu \mathrm{g} / \mathrm{mL})$ were irradiated by $808 \mathrm{~nm}\left(0.8 \mathrm{~W} / \mathrm{cm}^{2}\right)$ laser for $5 \mathrm{~min}$. After additional incubation for $24 \mathrm{~h}$, the medium was removed and cells were washed gently. Then cells were incubated with calcein-AM/PI for $30 \mathrm{~min}$ at room temperature, subsequently imaged by a NikonC1si laser scanning confocal microscopy. 
Cell apoptosis studies: Hela cells were incubated in a 24 -well plate for $24 \mathrm{~h}$ and then divided into eight groups. Following they were respectively treated with medium or COFs $(150 \mu \mathrm{g} / \mathrm{mL})$. After 6 hours of cultivation, they were irradiated by $808 \mathrm{~nm}$ $\left(0.8 \mathrm{~W} / \mathrm{cm}^{2}\right)$ laser for $5 \mathrm{~min}$. After additional incubation for $24 \mathrm{~h}$, the medium was removed and cells were moved away. Following removing the culture medium by washing with PBS for several times and the cells were digested by trypsin containing no EDTA. Eight groups of cells were respectively collected and stained by PI and annexin V-FITC to perform the cell apoptosis results.

In vivo antitumor activity and biosafety: Kunming strain mouse were obtained from the Hospital of Jilin University. After subcutaneously inoculated with U14 tumor cells and the tumors reached to a volume about $100 \mathrm{~mm}^{3}$, then all the mice were divided into four groups including PBS, PBS+Laser, DPPN COF, DPPN COF+Laser (four mice for each group). PBS or COFs $(4 \mathrm{mg} / \mathrm{mL}, 200 \mu \mathrm{L}$ for each mouse) were respectively intravenously injected into the corresponding group of mice. 12 hours later, two groups of mice were irradiated for 10 min with $808 \mathrm{~nm}(0.8$ $\mathrm{W} / \mathrm{cm}^{2}$ ) laser. Growth situation of mice, tumor weight and tumor volume were recorded every two days in two weeks. Then tumor tissue and major organs of the mice (heart, liver, spleen, lung and kidney) were collected for H\&E staining. To detect the biosafety of formulations, blood was collected for testing the level of serum alanine transaminase (ALT), aspartate transaminase (AST), uric acid (UA), urea (UREA) and creatinine (CREA). 


\section{Figures}

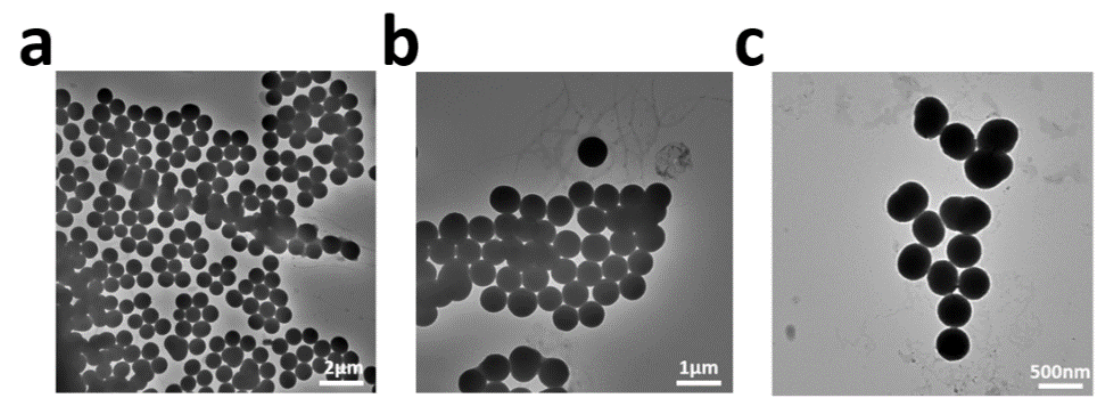

Figure S1. Transmission electron microscopy images of DPPN COF with different sizes $800 \mathrm{~nm}(\mathrm{a}), 600 \mathrm{~nm}(\mathrm{~b})$ and $400 \mathrm{~nm}(\mathrm{c})$.

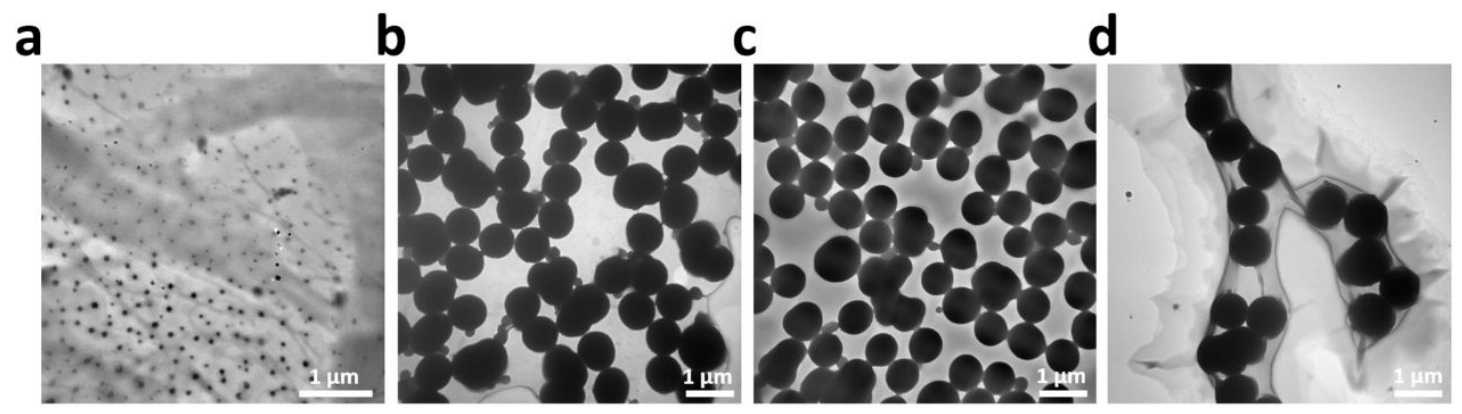

Figure S2. TEM images of DPPN COF reacted for $1 \mathrm{~h} \mathrm{(a),} 4 \mathrm{~h} \mathrm{(b),} 12 \mathrm{~h}$ (c) and $24 \mathrm{~h}$ (d).

a

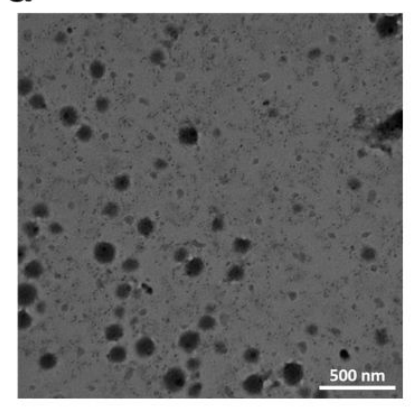

b

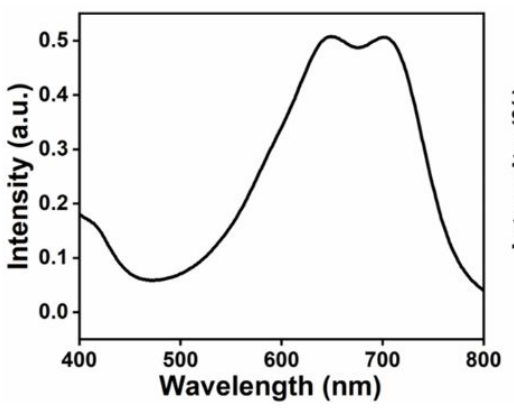

C

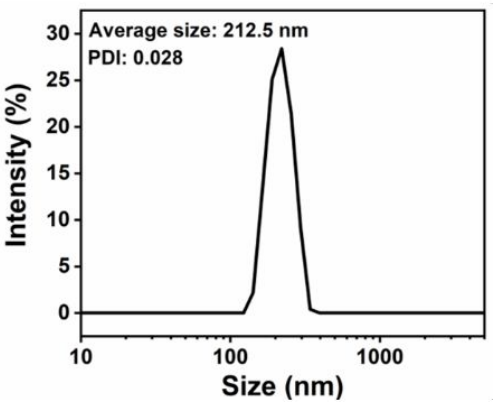


Figure S3. TEM images (a), UV absorption spectrum (b) and size distribution (c) of DPPN COF prepared in benzonitrile.
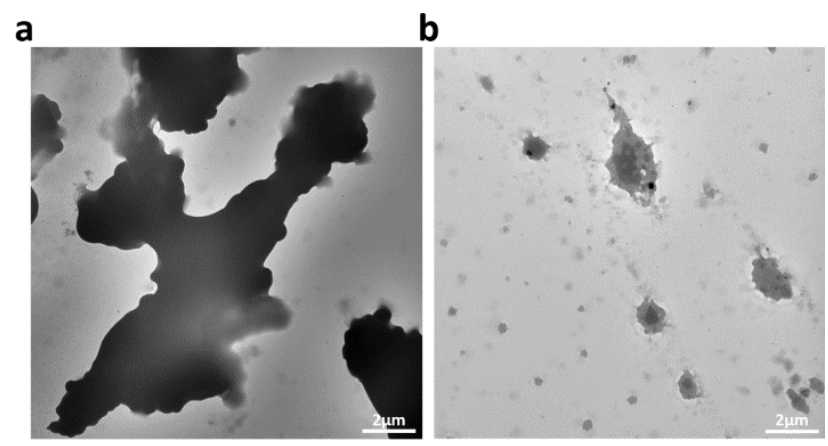

Figure S4. TEM images of DPPN COF using dioxane/ methanol (a) or acetone/ methanol (b) as solvents.

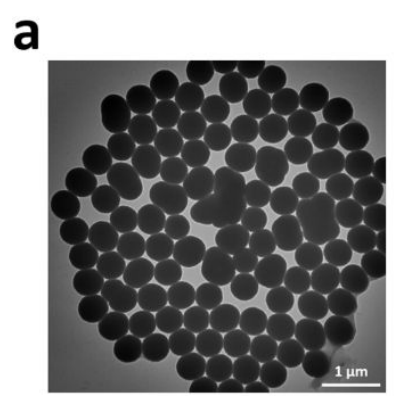

b

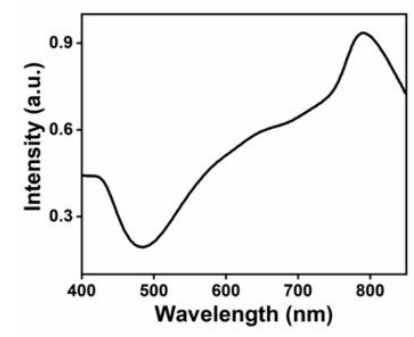

Figure S5. TEM images (a), UV absorption spectrum (b) of DPPN COF using chloroform/ethanol as solvent.
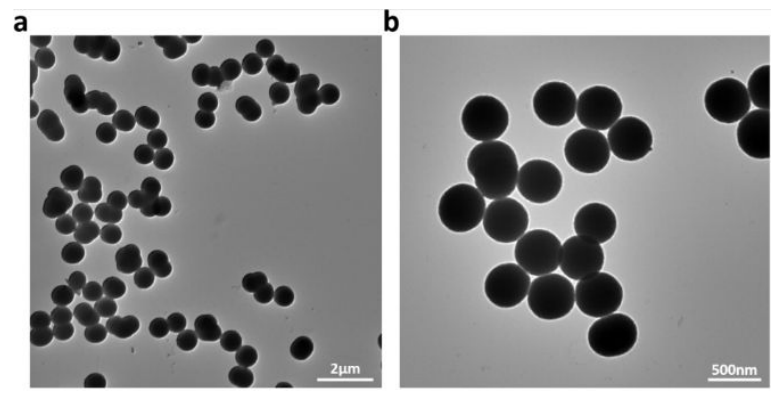

Figure S6. TEM images of DPPC COF with diameters of $800 \mathrm{~nm}$ (a) and $400 \mathrm{~nm}$ (b). 


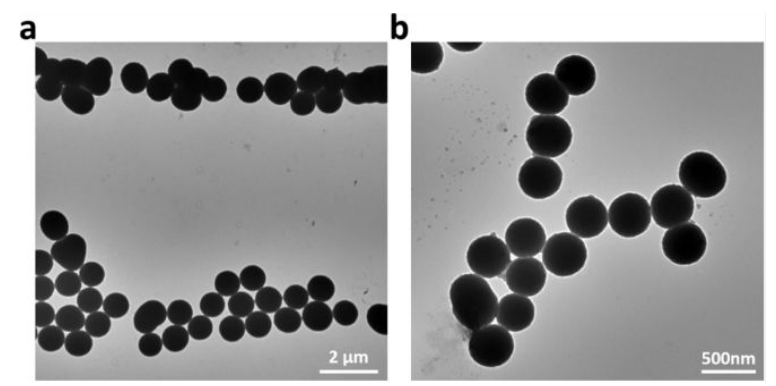

Figure S7. TEM images of DPPB COF with diameters of $800 \mathrm{~nm}$ (a) and $400 \mathrm{~nm}$ (b).

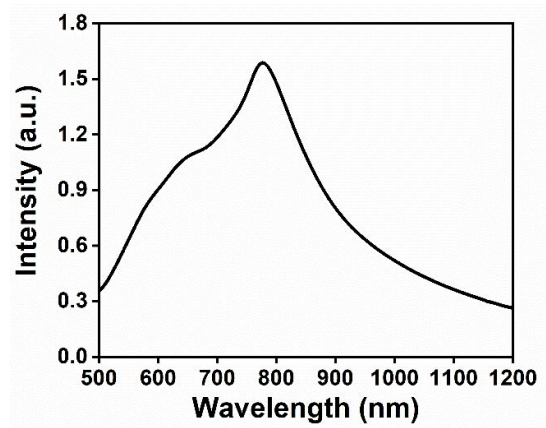

Figure S8. UV absorption spectra of DPPN COF from 500 to $1200 \mathrm{~nm}$ dispersed in THF.
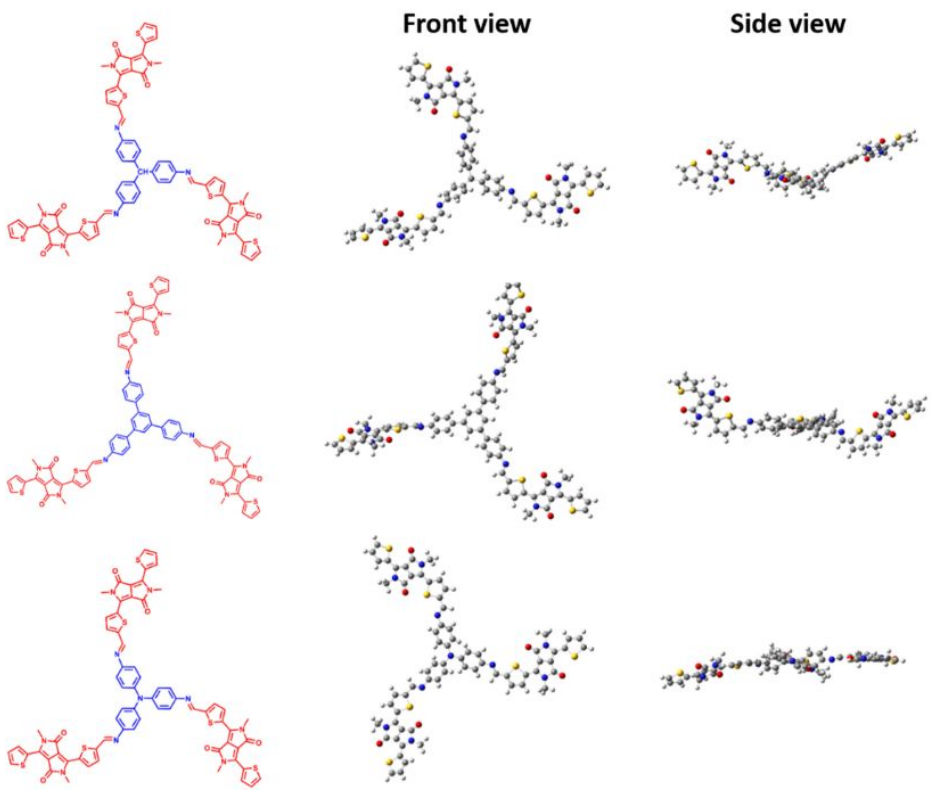

Figure S9. Schematic illustration of construction units and optimized spatial configurations of units of DPPC, DPPB and DPPN COF by DFT calculations. Carbon (grey), Nitrogen (blue), Oxygen (red) and Hydrogen (white). 


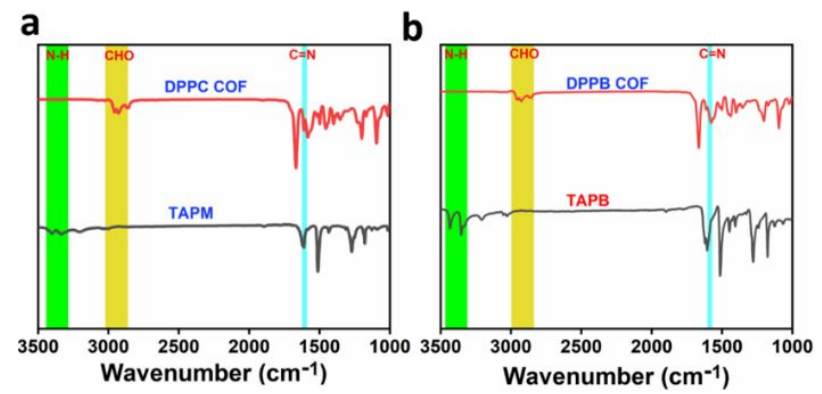

Figure S10. FT-IR spectra of DPPC COF (a) and DPPB COF (b) and their corresponding building blocks.

a

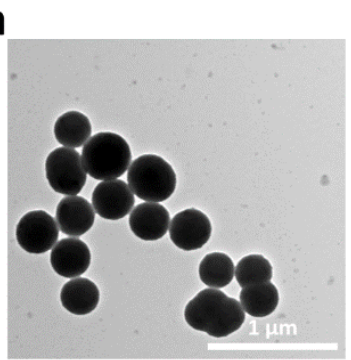

b

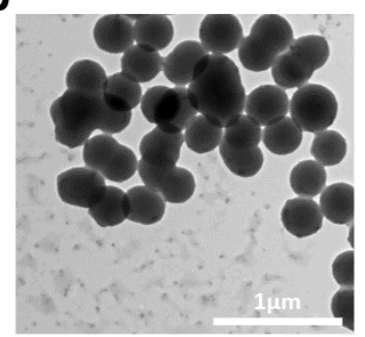

Figure S11. Transmission electron microscopy images of DPPN COF with $300 \mathrm{~nm}$ diameter dispersed in THF (a). Transmission electron microscopy images of DPPN COF with $300 \mathrm{~nm}$ diameter assembled with $\mathrm{MPEG}_{2000}$-DSPE in water (b).

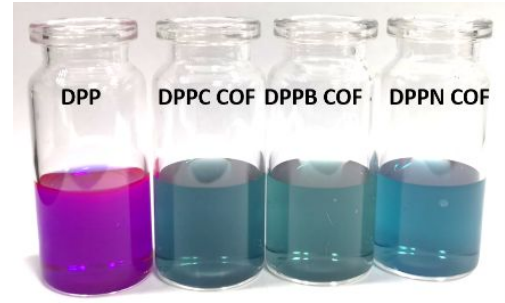

Figure S12. Images of DPP monomer dissolved in THF and COFs dispersed in THF.

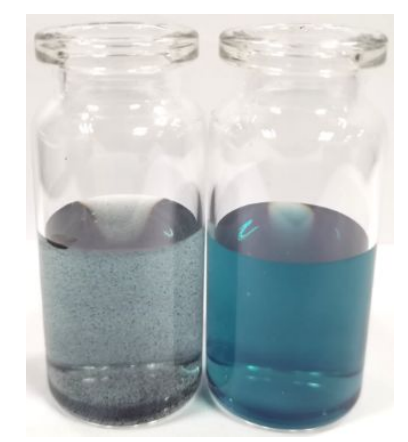

Figure S13. Images of DPPN COF before (left) or after (right) assembled with $\mathrm{MPEG}_{2000}$-DSPE dispersed in water. 


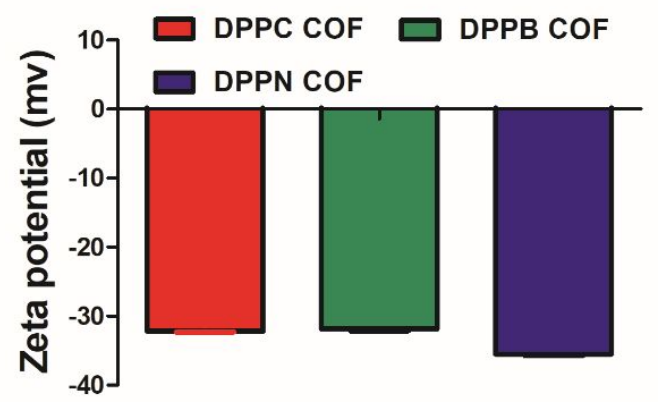

Figure S14. Zeta potential of DPPC, DPPB and DPPN COF dispersed in water.

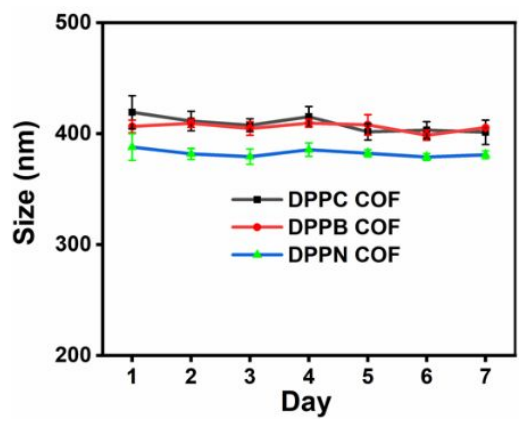

Figure S15. Colloidal stability of COFs dispersed in water for 7 days.

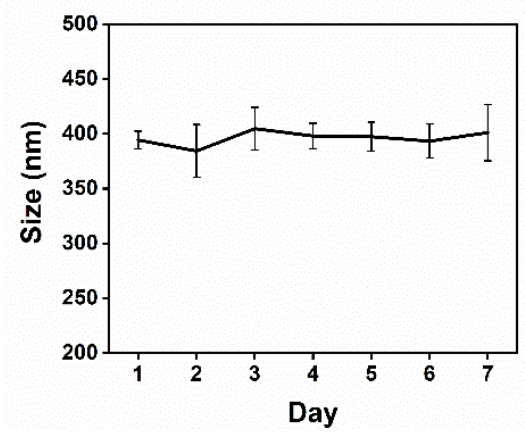

Figure S16. Colloidal stability of COFs dispersed in pH 6.5 PBS for 7 days.

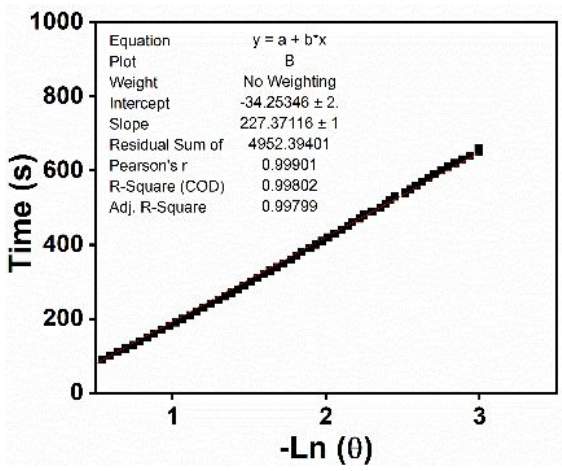


Figure S17. The relationship between cooling period of time and the negative natural logarithm of temperature of DPPN COF.
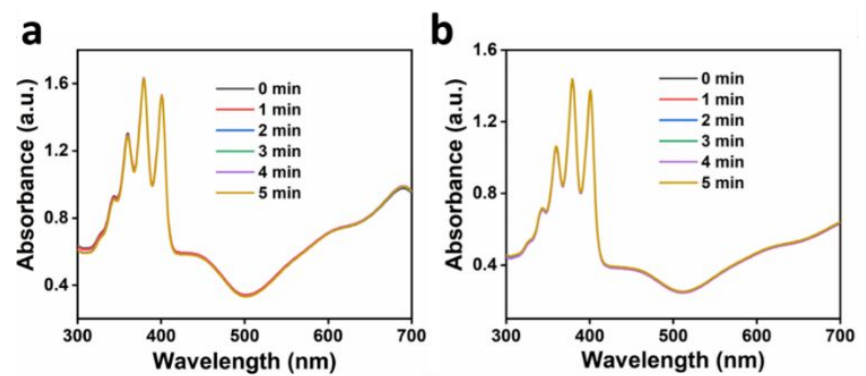

Figure S18. Photodynamic effect of DPPC COF (a) and DPPB COF (b) detected by ABDA under $808 \mathrm{~nm}$ laser irradiation.

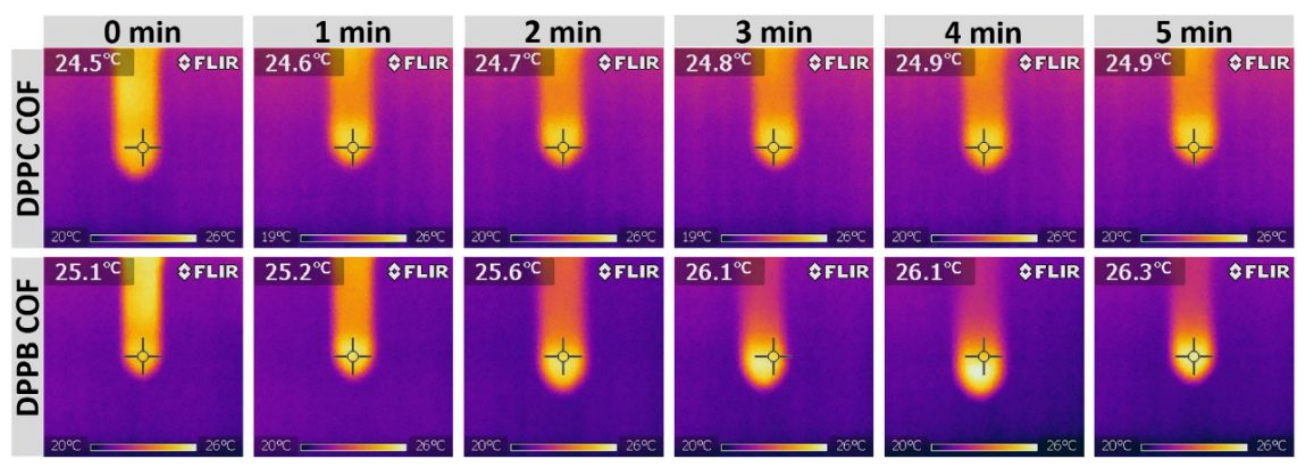

Figure S19. Thermal images of DPPC COF and DPPB COF with $808 \mathrm{~nm}$ laser irradiation at an intensity of $1 \mathrm{~W} / \mathrm{cm}^{2}$ in $5 \mathrm{~min}$.
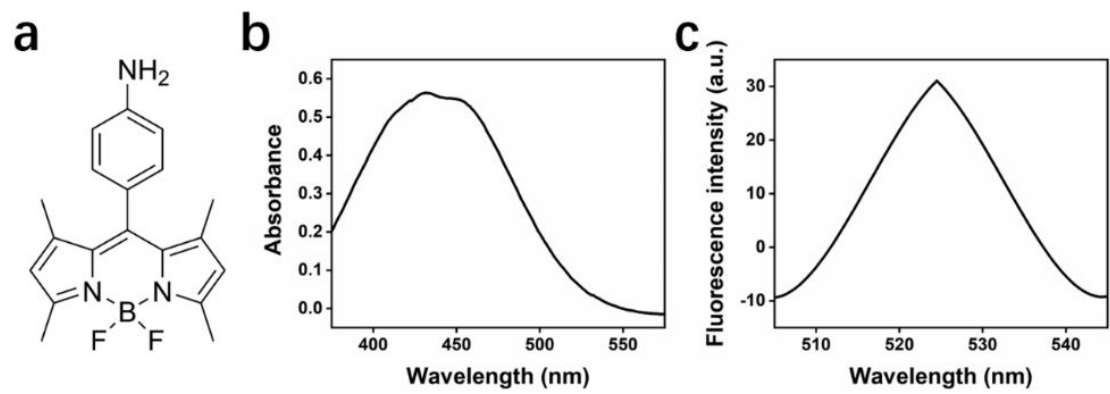

Figure S20. Molecular structure, UV absorbance spectrum and fluorescence spectrum of BDP with green fluorescence (Excited by $450 \mathrm{~nm}$ wavelength). 
a

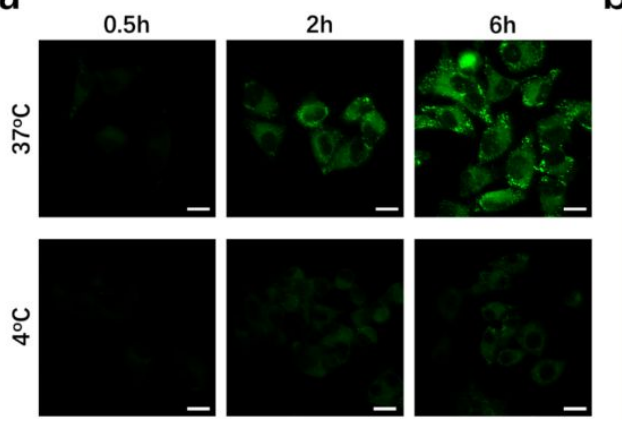

b

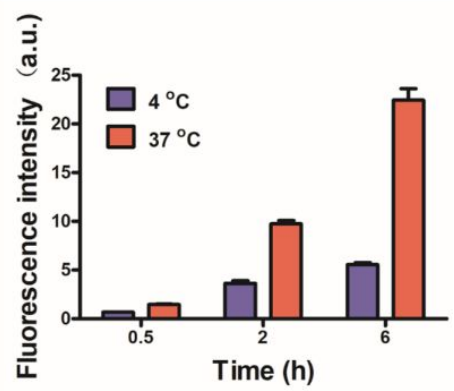

Figure S21. Endocytosis contrast of DPPN COF/BDP at different time points and temperature (a). Fluorescence intensity comparison of DPPN COF/BDP (b). Scale bars, $20 \mu \mathrm{m}$.
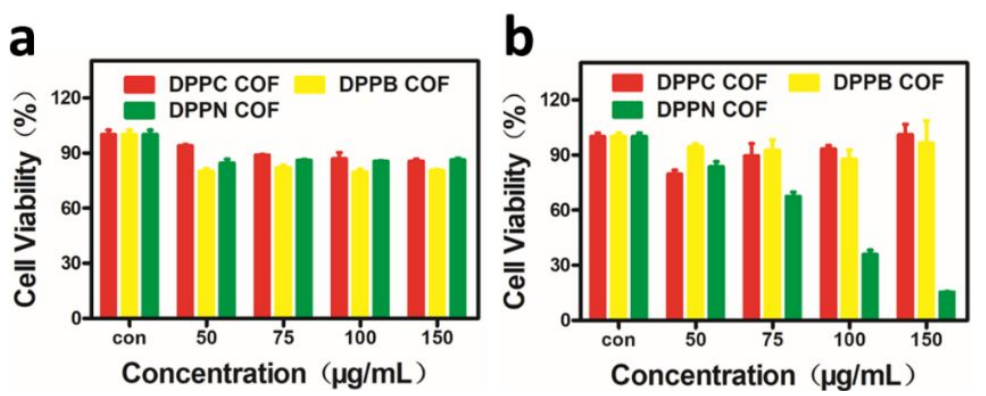

Figure S22. Cytotoxicity of A549 cells (a) treated with DPPC, DPPB, and DPPN COF without laser irradiation. Cytotoxicity of A549 cells (b) and treated with DPPC, DPPB, and DPPN COF with $808 \mathrm{~nm}$ laser $\left(0.8 \mathrm{~W} / \mathrm{cm}^{2}\right)$ irradiation for $5 \mathrm{~min}$.

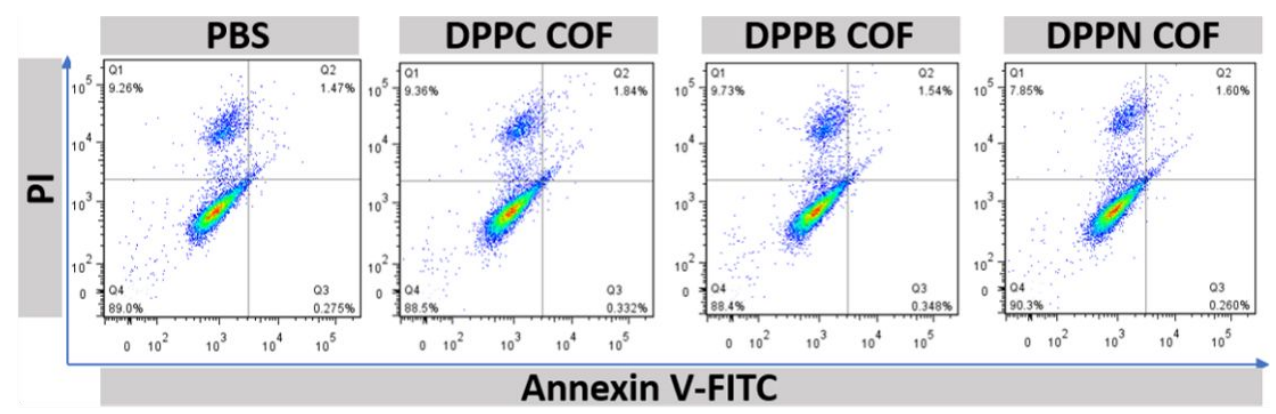

Figure S23. Cell apoptosis assay of HeLa cells treated with PBS or COFs without laser irradiation. 
a

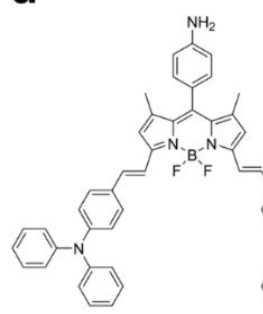

b

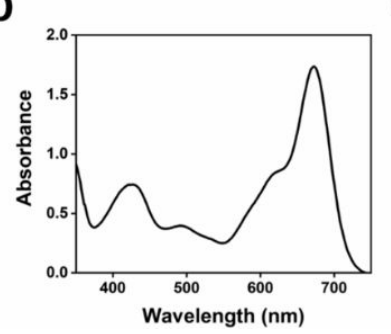

C

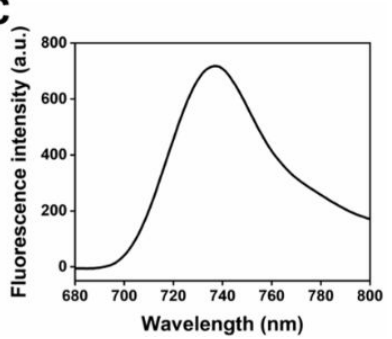

Figure S24. Molecular structure (a), UV absorbance spectrum (b) and fluorescence spectrum (c) of NIR BDP with red fluorescence (Excited by $670 \mathrm{~nm}$ wavelength).

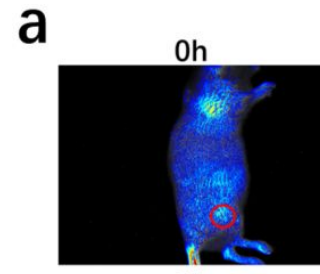

$6 \mathrm{~h}$

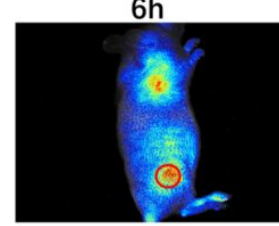

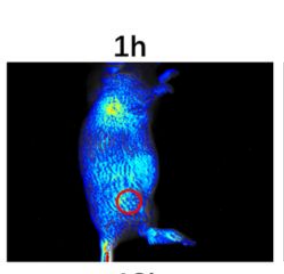

$12 \mathrm{~h}$

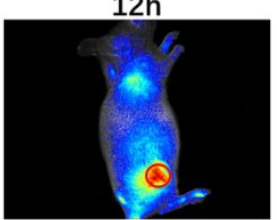

$3 h$

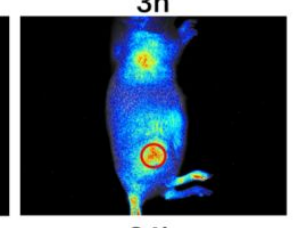

$24 \mathrm{~h}$

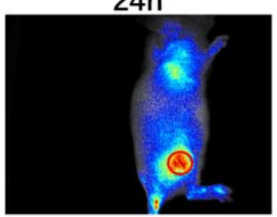

b

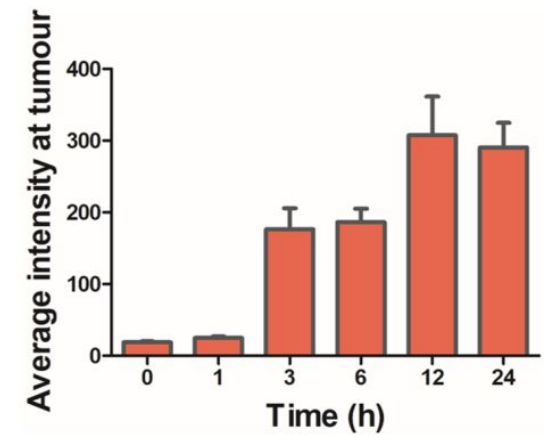

Figure S25. Fluorescence imaging of mice after intravenous injection from 0 to $24 \mathrm{~h}$, tumors were circled (a). Fluorescence intensity statistics of mice at different points in time (b).

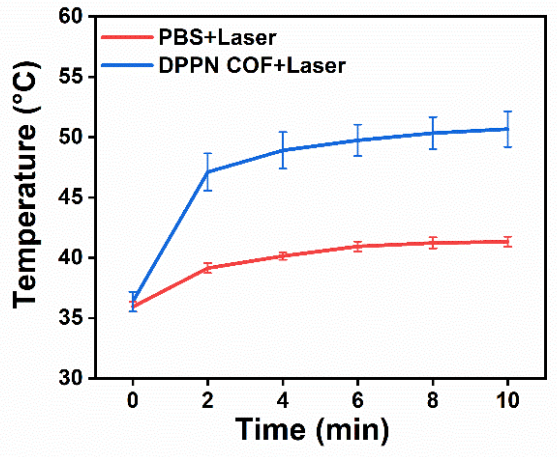

Figure S26. Temperature plots of two groups of mice with laser irradiation for $10 \mathrm{~min}$ at different point of time. 


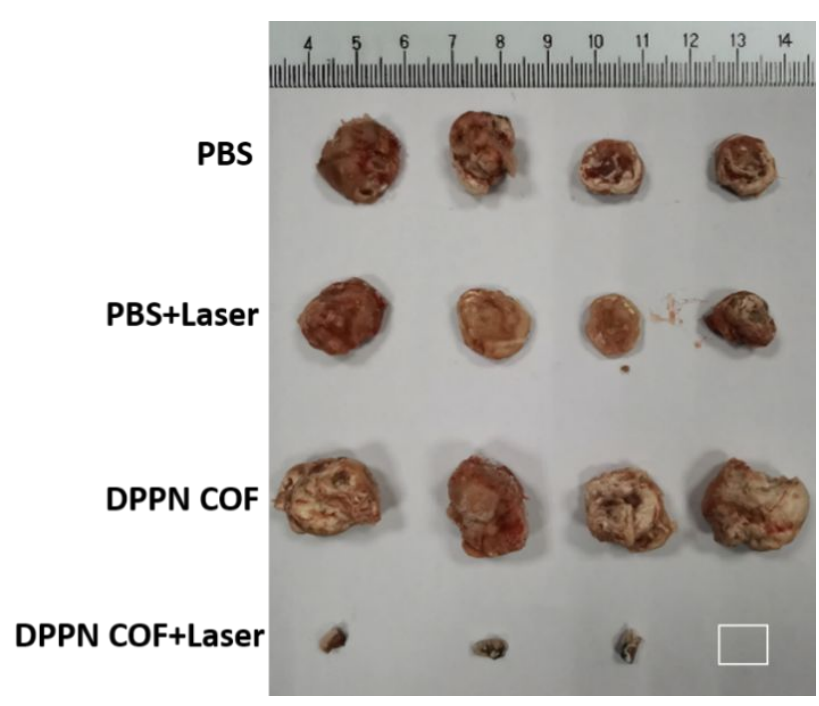

Figure S27. Corresponding images of tumors of four groups of mice after treatment for 14 days, $n=4$.

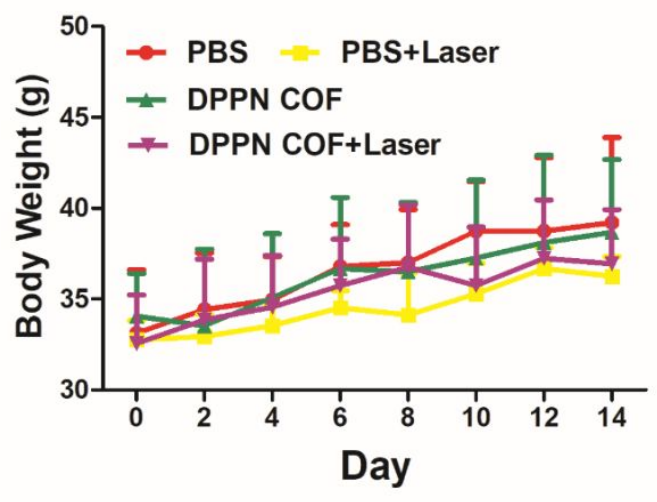

Figure S28. The corresponding weight changes of four groups of mice in 14 days.
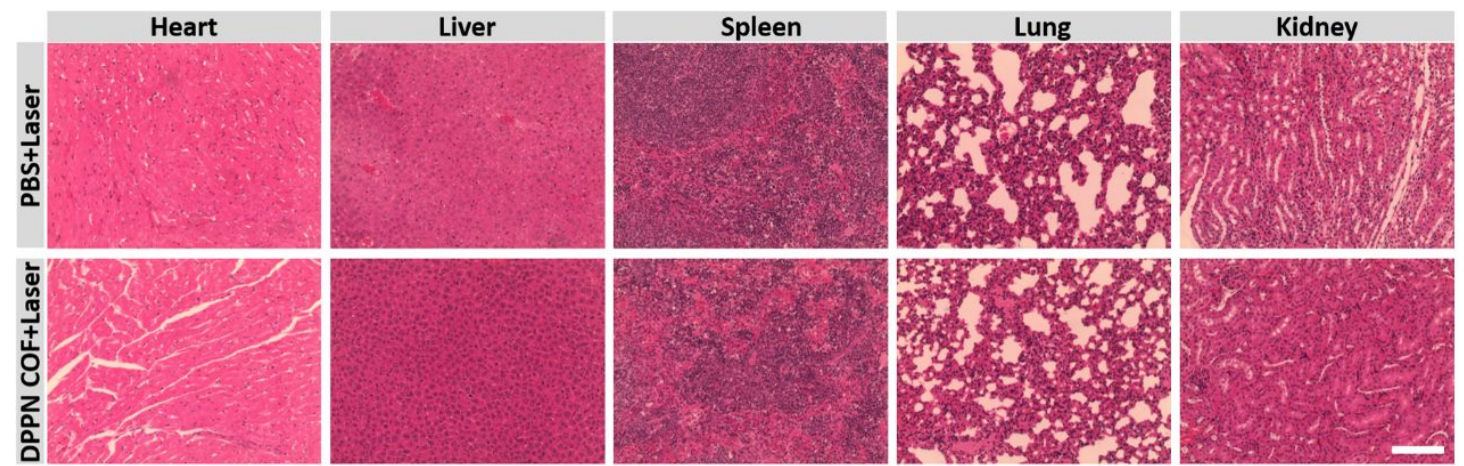

Figure S29. H\&E staining of different organs of groups of mice with laser irradiation. Scale bars, $100 \mu \mathrm{m}$. 

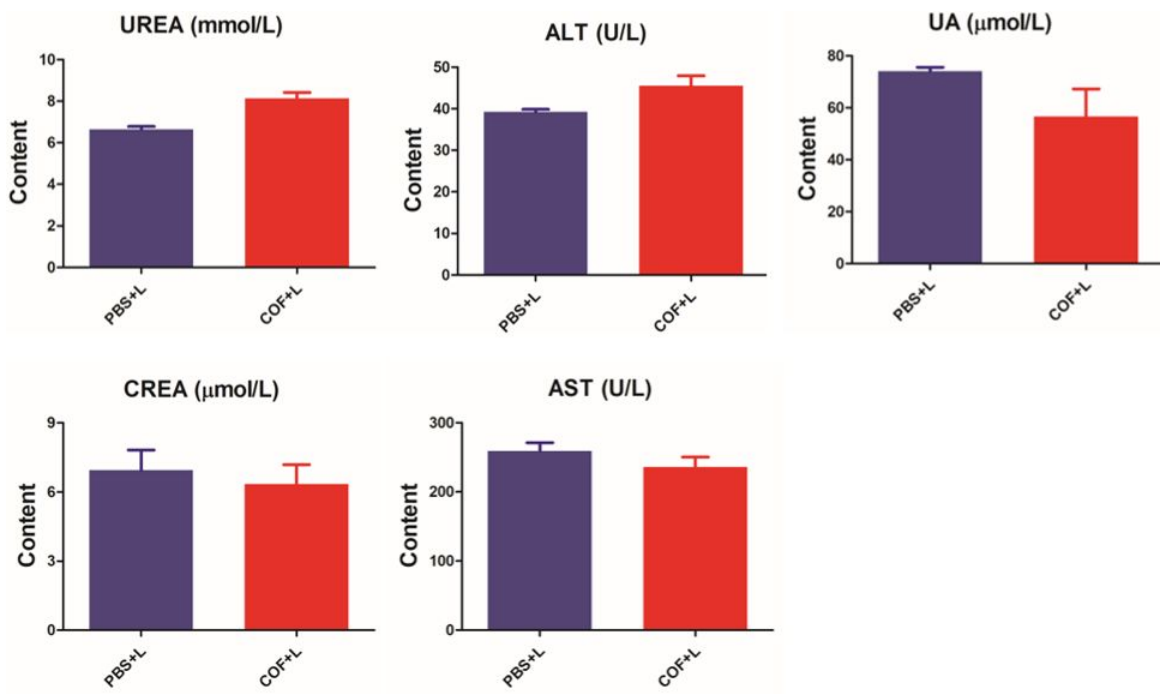

Figure S30. UREA, ALT, UA, CREA and AST levels in the blood of groups of mice with laser irradiation.

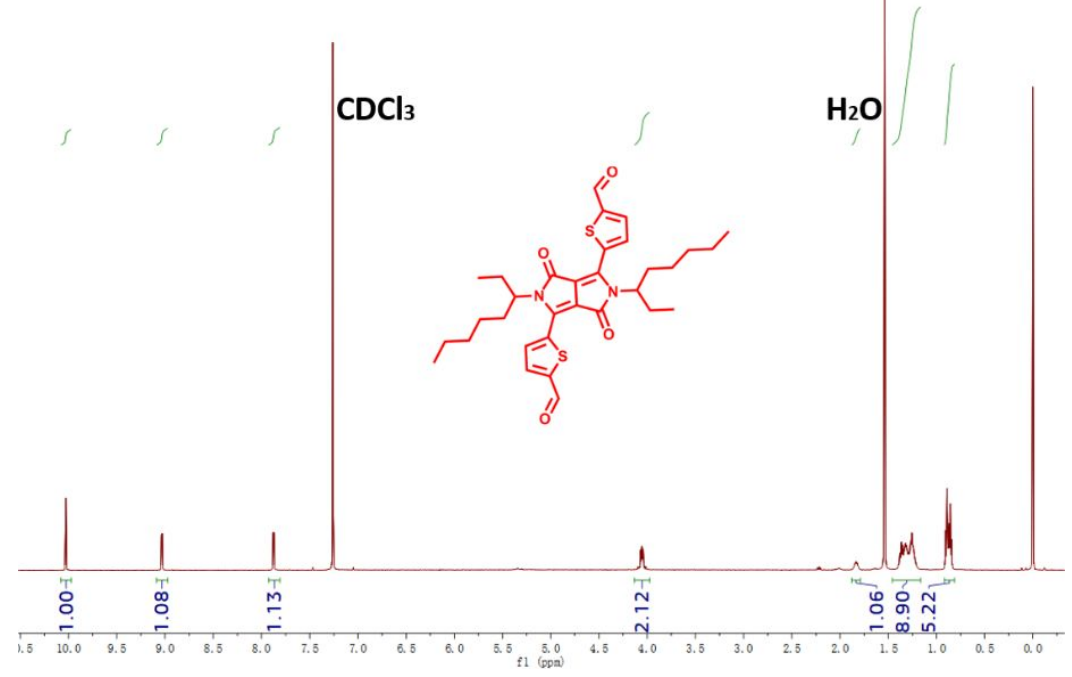

Figure S31. ${ }^{1} \mathrm{H}$ NMR spectrum of DPP in $\mathrm{CDCl}_{3}$.

\begin{tabular}{|c|c|c|c|}
\hline & DPPC COF & DPPB COF & DPPN COF \\
\hline Z-Average $(\mathbf{n m})$ & 415.6 & 400.4 & 379.9 \\
\hline PDI & 0.017 & 0.034 & 0.038 \\
\hline
\end{tabular}

Table S1. Hydrodynamic size and polydispersity index (PDI) of DPPC, DPPB and DPPN COF dispersed in water.

\section{References}


(1) Zheng, X.; Wang, L.; Liu, S.; Zhang, W.; Liu, F.; Xie, Z. Nanoparticles of Chlorin Dimer with Enhanced Absorbance for Photoacoustic Imaging and Phototherapy. Adv. Funct. Mater. 2018, 28 (26), 1706507. 